\title{
La Ética de Immanuel Kant
}

The Ethics of Immanuel Kant

\author{
Ana María Fajardo Fajardo ${ }^{1}$ \\ Universidad Externado de Colombia
}

Recibido: 04.11.2020

Aceptado: 18.11.2020

\section{Resumen}

Kant, al analizar las éticas anteriores a la suya, nota que no lo satisfacen lo suficiente, por razón de fundamentar la moral en principios contingentes, o en la antropología, como dice él mismo, es decir, en las inclinaciones de la naturaleza humana. Entre estos tipos de éticas, distintas a la de Kant, tenemos la moral religiosa, que pone su razón de ser en la voluntad de Dios y en la salvación del alma; promulgando de esta manera premios para quienes viven lo mandado por Dios y castigos para quienes no cumplen sus leyes; la visión aristotélica, cuya finalidad es promover la felicidad; y la sentimentalista, que se atiene a la experiencia de placer o dolor. La característica de estos planteamientos consiste en que consideran la ética bajo un contenido.

Palabras clave: Ciencias de la Educación, Filosofía, Ética, Lógica, Naturaleza humana, Ontología, Existencialismo.

\footnotetext{
1 anafajardo03@est.uexternado.edu.co https://orcid.org/0000-0001-8103-8382

https://scholar.google.com/citations?user=gcqQvF8AAAAJ\&hl=es\&oi=ao
} 


\begin{abstract}
Kant, when analyzing ethics prior to his, notes that they do not satisfy him sufficiently, by reason of grounding morality on contingent principles, or on anthropology, as he himself says, that is, on the inclinations of human nature. Among these types of ethics, different from Kant's, we have religious morality, which puts its reason for being in the will of God and in the salvation of the soul, thus promulgating prizes for those who live what God has commanded and punishments for those who do not comply with its laws; the Aristotelian vision, whose purpose is to promote happiness; and the sentimentalist, who sticks to the experience of pleasure or pain. The characteristic of these approaches is that they consider ethics under a content.
\end{abstract}

Keywords: Education Sciences, Philosophy, Ethics, Logic, Human nature, Ontology, Existentialism.

El libro de Inmanuel Kant titulado "Fundamentación de la metafísica de las costumbres", publicado en el año de 1785, es un trabajo sobre la filosofía de la moral (García San Miguel, 2006). A lo largo del texto el autor busca desarrollar el sistema de toda la moralidad y determinar toda la estructura sobre la que puede construirse (Carlos Andrés Moreno Urán; Katherine Esponda Contreras, 2008). Kant propone acercarse al principio fundamental de la moralidad y criticar su posibilidad (Castro, 2004). Es un texto en la misma línea con su temática de la Crítica de la razón práctica, aunque más accesible.

El libro tiene los siguientes capítulos:

Prólogo.

Tránsito del conocimiento moral vulgar de la razón al conocimiento filosófico.

Tránsito de la filosofía moral popular a la metafísica de las costumbres.

Paso final de la metafísica de las costumbres a la crítica de la razón pura práctica. 
Entre estas éticas de contenidos hay algunas diferencias importantes (Casales García, 2014). La visión aristotélica, aunque se guía por parámetros de racionalidad -igual que los planteamientos kantianos- no es de carácter formal sino de contenidos. Dicha concepción se propone fines y, en vista a sus fines, determina el valor moral de la acción (Román Maestre, 2007); su característica fundamental consiste en que funda sus principios en la razón, a diferencia de la ética empírica que los funda en sentimientos. Pero como lo decíamos, aunque sus principios son racionales, el valor moral de la acción se juzga de acuerdo con el fin propuesto (Habermas, 1997). La moral de corte religioso tiene su punto de partida en la fe este es el caso del cristianismo-, se sustenta en la revelación; el valor moral de una acción está de acuerdo con el grado de respeto que se tenga a la voluntad de Dios. La ética empírica en cambio pone el énfasis en el sentimiento; para esta postura, la moralidad es el desarrollo natural de ciertos sentimientos que pertenecen a nuestra naturaleza humana. Veamos esto con un poco más de detalle.

La ética empírica pone el énfasis en el sentimiento (Rivera Castro, 2004), la acción moral se juzga de acuerdo con el grado de felicidad, satisfacción o bienestar que produce una acción. En la antigüedad dicha visión - corriente hedonista- era expuesta por Aristipo de Cirene y Epicuro, aunque este último se inclinaba no sólo por los placeres del cuerpo, pues incluía también los placeres intelectuales. Precisamente ha sido en los siglos XVII y XVIII la gran época de auge de la ética empírica desarrollada por los ingleses, especialmente por F. Hutcheson (1694-1746) y D. Hume (1711-1776), posición que se ha dado en llamar "moral del sentimiento", la cual conocía muy bien nuestro filósofo de Königsberg.

Cabe señalar que la moral expuesta por estos dos autores no se reducía al simple sentimiento egoísta (Gachama Muñoz, 2010); se orientaba hacia sentimientos morales de benevolencia, simpatía, sinceridad y comprensión hacia los hombres, con el fin de contribuir en la promoción de la felicidad propia y de los demás. Como se ve, ésta es una postura de dignos ideales a seguir; entonces, ¿en qué estaba fallando este tipo de ética, para que fuese rechazada por Kant? La razón principal de dicho rechazo vendría a ser lo siguiente: 
La ética empírica niega el sentido trascendente de la autodeterminación desde la libertad, es decir, desde el hacerse práctica la razón pura por sí misma. Así pues, el empirismo reduce la praxis humana a la motivación de las inclinaciones de placer o dolor, esto es a la sensibilidad. (Anzenbacher, 1993, p. 90)

Precisamente el punto débil de esta visión es que nada tiene que ver con el hacerse práctica la razón (Mockus Sivickas, 1994). La posición empírica se opone a la autodeterminación y nos encierra en la causalidad natural, esto es, en la inclinación; si no somos libres, entonces no podemos autodeterminarnos, pues nuestra voluntad obedecería a los mandatos de la inclinación - placer/dolor- y no de la razón. La razón pura se hace práctica cuando se presenta como fundamento de determinación suficiente de la voluntad, independiente de los motivos empíricos de placer y dolor. Si esto resulta cierto, entonces quedaría mostrado que la libertad se halla por encima de la causalidad del mundo natural (Arteta Ripoll, 2016), es decir, que sería posible autodeterminarnos, y no quedaríamos presos en los motivos de nuestra inclinación, en tanto que la moral es posible justificarla a priori.

Brevemente podemos decir que por a priori entiende Kant todo conocimiento que es independiente de la experiencia, de las impresiones de los sentidos; este tipo de conocimiento se caracteriza por incluir leyes lógicas que valen universalmente para todo ser racional. La ley moral es de este tipo, se asienta en conceptos puros de la razón, en una metafísica (Cordini, 2018). La moral kantiana se caracteriza por valerse o apoyarse en conceptos racionales, objetivos, universales, es una metodología netamente a priori. Al tiempo que presenta una gran novedad, es el punto central desde donde se aborda dicha postura moral. Esta característica a priori se encuentra expuesta en la obra de la Fundamentación, y con más claridad y propiedad en la segunda crítica. Este asunto de la fundamentación podemos expresarlo a partir de los siguientes interrogantes: ¿Cómo construir una ética a priori, ¿cuáles son sus características, ¿cómo justificar a priori la moral? La cuestión de la fundamentación de la moral no es una problemática nueva; a la respuesta de ¿dónde justificar los juicios morales? unos habían contestado que en los fines que se persiguen -felicidad, placer-; desde 
esta perspectiva una acción sería buena en la medida que concordara con el fin propuesto (Casales-García, 2011). Otros decían que en la revelación -moral religiosa, específicamente de corte cristiano- una acción era moral si estaba de acuerdo con la voluntad de Dios. La ética empírica por su parte niega toda posibilidad de buscar los principios de la acción en la razón pura, y nos somete a la determinación de nuestras inclinaciones (Dasuki, 2010). Kant rehusó los presupuestos empíricos, en tanto que no son compatibles con el carácter racional y objetivo del valor moral de una acción.

A la cuestión de la fundamentación de los principios morales, Kant responde con su apriorismo; como ya lo anotábamos, en su obra -Fundamentación de la Metafísica de las Costumbres-, concretamente en la segunda sección, el filósofo se refiere a este asunto con el fin justificar el concepto de deber moral (Gonzalez Vallejos, 2012); allí insistirá en las características y necesidad de una moral a priori.

Esta temática, la desarrollará el autor con más amplitud en la Crítica de la razón práctica; Es decir, este asunto del carácter a priori de la moral lo desarrollará el autor propiamente y con más claridad en una obra posterior, la Crítica de la razón práctica. Esta obra consta de un prólogo y una introducción, en los que se plantea el problema propio del escrito, y dos partes: una analítica, en la que se analizan los principios a priori prácticos, y una dialéctica, en la que se analiza y resuelve la antinomia de la razón práctica. Esta obra no tiene la parte de la estética, ya que la razón pura práctica procede al margen de la experiencia (Colomer, 2006).

La moral, afirma Kant en el prólogo de la Fundamentación, ha de mantenerse libre de todo contacto con la experiencia si quiere validez universal y objetiva; de ahí la siguiente formulación que propone el autor: “¿No se cree que sea de la más urgente necesidad elaborar de una vez por toda una filosofía moral pura que esté completamente limpia de todo cuanto pueda ser empírico y pertenezca a la antropología?” (Kant, 1985, p. 49).

Con esta formulación el filósofo alude a la necesidad de pensar la moral a partir de conceptos originados exclusivamente en la razón (Aparicio-Gómez, 2019). Por el carácter de 
universalidad y necesidad - propuestos por Kant- los principios de la moral han de buscarse $a$ priori. En el capítulo segundo de su obra para referirse al concepto de deber, enfatiza en el hecho de concebir la moral a partir de características a priori:

No hay más que mirar los ensayos sobre la moralidad que se han escrito según los gustos de esta época, y se verá enseguida cómo se mezclan en extraño consorcio, ya la peculiar determinación de la naturaleza humana, -comprendida en ella también la idea de una naturaleza racional en general-, ya la perfección, ya la felicidad, aquí el sentimiento moral, allí el temor de Dios, un poco de esto, un poco de aquello..., sin que a nadie se le ocurra preguntar si los principios de la moralidad han de buscarse en el conocimiento de la naturaleza humana, -que no podemos obtener más que por medio de la experiencia- o si en el caso de que la respuesta sea negativa, deben buscarse en los conceptos absolutamente puros de la razón, libres de todo cuanto sea empírico, y completamente a priori. (Kant, 1985, p. 66)

Al afirmar Kant, que todos los conceptos éticos tienen su origen en la razón totalmente $a$ priori, niega la tesis del empirismo ético según la cual se obra siguiendo los sentimientos. La experiencia no puede dar lugar a leyes universales y necesarias; en consecuencia, para conceder objetividad a la acción ésta ha de ser a priori, ya que ha de valer para todos los seres racionales (Aramayo, 2012). Dicho en otras palabras, si la razón determina a priori la acción de la voluntad, quiere decir entonces que la motivación de agrado o desagrado no tiene cabida en la determinación de los principios de la moralidad.

En fin, una fundamentación moral no enraizada en planteamientos pragmáticos, o en la antropología, tiene que ir irremediablemente a parar en una metafísica (Aparicio-Gómez, 2006). Si queremos salir de nuestro estado de infantilismo, narcisismo, egoísmo, sentimentalismo, es obvio que no se puede fundamentar la moral en presupuestos empíricos o religiosos, ha de fundamentarse a priori. 
En la brillante introducción que hace Luis Martínez de Velasco a la Fundamentación, recoge con suma claridad la cuestión de lo a priori y el anclaje motivacional, temas centrales en la reflexión moral de Kant:

La estricta auto fundamentación de una moral racional se basa en la vinculación conceptual establecida a priori entre la conciencia pura del deber como un factum (un hecho dado) de la razón moral y la madurez ética del género humano, como resultado de un proceso de auto-objetivación capaz de desarrollarlo en toda su racionalidad. (Kant, 1985, p. 23)

Podemos rescatar de este párrafo dos ideas pertinentes: el a priori moral como un hecho dado, y el asunto del anclaje motivacional o la vinculación de lo teórico y lo práctico (AparicioGómez, 2016). El a priori moral como un hecho dado vendría a significar una realidad que está presente y no necesita de justificación; esto quiere decir que no podemos no ser morales; tenemos que serlo debido a nuestra condición de seres humanos (Mauricio, 2007). La moralidad desde la perspectiva del a priori no es un invento de la razón; ella está encargada simplemente de señalar el carácter de nuestros conceptos morales, más no de demostrar la aprioridad de sus conceptos, ya que éstos no necesitan demostración. El carácter objetivo de este hecho no puede deducirse teóricamente, ni ser confirmado por la experiencia.

La segunda idea hace referencia al anclaje motivacional, ¿cómo hacer posibles, o accesibles los postulados racionales -el carácter a priori- al hombre concreto? Ya que la realidad moral no es una construcción exclusiva para un grupo de eruditos o de filósofos, pues ésta pertenece a todo hombre, cualquiera que sea su condición. En este mismo sentido, ¿cuál puede ser el móvil que impulse a la voluntad a cumplir con la ley moral? En el intento de lograr este objetivo, Kant presenta la voluntad humana en términos de sometimiento o auto sometimiento a dicha ley. Para lograr esta meta se requiere un proceso de maduración que nos vincula con el sentido de aceptar el mandato que la razón impone a la voluntad, aceptación que sólo puede ser posible por la vía de la autonomía. 
Aunque los principios morales se han de fundamentar a priori, ha de ser posible derivar de ellos reglas prácticas para toda naturaleza humana. En definitiva, el carácter apriorístico es para Kant la garantía de validez de su postura moral.

Queda abierto así el interrogante: ¿Por qué la moral debe ser a priori? ¿Por qué Kant insiste en su deseo de certeza, de objetividad, quién se la está pidiendo? El argumento que nos ofrece Kant enfatiza el hecho de que los postulados de la razón, si han de valer para todos, tienen que ser objetivos y cumplir con el elemento de la universalidad; por ello sólo pueden valer a priori. Nos parece que dicho argumento no responde propiamente por la razón del interrogante, sino por sus características.

Como lo anotábamos anteriormente, la aprioridad es un hecho que no necesita demostración; es decir, el uso del concepto de demostración se puede tomar en distintos sentidos: como una prueba física de hechos empíricos, o de hechos psicológicos. Cuando decimos que Kant no demuestra la libertad o el a priori de su moral, lo tomamos en sentido empírico; en otras palabras, la experiencia no nos sirve para demostrar ni la libertad, ni el a priori ético (Norbert, 1992). En su obra posterior, la Crítica de la razón práctica, el autor señalará efectivamente que el carácter de la moral a priori queda demostrado por el hecho de haber encontrado el imperativo categórico, y la autonomía como principio a priori de determinación de la voluntad. La demostración que realiza Kant es de tipo trascendental, no de tipo empírica, es decir, que una demostración de carácter trascendental vale para todos los seres racionales. Este es precisamente el sentido que le da nuestro filósofo al concepto de demostración. es el supuesto de la moralidad. En consecuencia, lo que hay que hacer es señalar sus características, es decir, su universalidad y necesidad junto con la pureza de sus conceptos, que son fruto de la razón y no de la experiencia.

Podríamos afirmar, por último, que el espejo de la moral racional pura está caracterizado por la reflexión y el método que las ciencias exactas han llevado a cabo, lo cual Kant conoce perfectamente -las matemáticas y la física-, similitud que ha sido incorporada por el autor en su propuesta moral. El modelo de la ciencia moderna ha mostrado que la razón es la que 
determina a la naturaleza según leyes; pues bien, así debe suceder con la moralidad: los principios de la conducta han de buscarse nada más que en la razón.

Precisamente aquí se vincula el principio de la autonomía, que consiste en elegir siempre de tal modo que las máximas de nuestra elección puedan ser comprendidas como leyes universales. Desde la autonomía de la voluntad cada sujeto se impone a sí mismo la ley, que es válida para todo ser racional (Placencia, 2013). No se podría considerar que un ser humano es autónomo, cuando se deja llevar por la inclinación, o por el agrado o desagrado que una determinada acción le produce, en tanto que estaría rompiendo con el carácter objetivo de la moral.

\section{Conclusiones}

Tenemos dos ideas centrales en este apartado, al cual le hemos dado el nombre de característica fundamental de la obra.

Una idea se refiere al carácter de la fundamentación a priori; la moral según Kant se funda en conceptos puros de la razón, libres de todo cuanto sea empírico.

Otra idea alude al hecho de que la moral kantiana se sostiene o tiene validez sólo a partir del principio de la autonomía. La autonomía se deriva del análisis de la moralidad, en cuanto implica leyes prácticas objetivas, esto es universales y necesarias.

\section{Referencias}

Anzenbacher, Arno (1993). Introducción a la filosofía. Madrid. Herder.

Aparicio-Gómez, Oscar-Yecid (2006). El nexo familia persona en la filosofía personalista (Tesis Doctoral). Barcelona, España: Universidad de Barcelona. 
Aparicio-Gómez, Oscar-Yecid (2016). La persona en la Sociedad Digital. En AparicioGómez, Oscar-Yecid (Eds.). El Uso Educativo de las TIC (pp. 251-256). Bogotá, Colombia: Universidad Central. ISBN: 978-958-26-0316-8

Aparicio-Gómez, William-Oswaldo (2019). The community dynamics as a divine pedagogy. En: Revista Interamericana de Investigación, Educación y Pedagogía, RIIEP. Vol. 12, Núm. 1. p. 55 - 71. ISSN: 1657-107X - e-ISSN: 2500-5421. DOI: 10.15332/s1657107X.2019.0001.08

Aramayo, R. R. (2012). Estudio preliminar. El empeño kantiano por explorar los últimos confines de la razón. In Fundamentación para una metafísica de las costumbres.

Arteta Ripoll, C. (2016). Sobre ética y derecho en la filosofía de Kant. Amauta. https://doi.org/10.15648/am.27.2016.6

Casales-García, R. (2011). Estructura de la filosofía práctica de Kant. (Spanish). The Structure of Kant's Practical Philosophy. (English).

Casales García, R. (2014). La división de la filosofía práctica en Kant. Revista Valenciana, Estudios de Filosofía y Letras. https://doi.org/10.15174/rv.v0i15.72

Castro, F. R. (2004). El Imperativo Categórico en la Fundamentación de la Metafísica de las costumbres. Revista Digital Universitaria.

Colomer, Eusebi. (2006). El pensamiento alemán de Kant a Heidegger. Tomo I. La filosofía trascendental: Kant. Madrid. Herder

Cordini, N. S. (2018). La fundamentación y finalidad de la pena en las "Lecciones sobre filosofía moral": Un estudio sobre el pensamiento kantiano previo a "La metafísica de las costumbres.” Revista Electrónica Instituto de Investigaciones Jurídicas y Sociales Ambrosio L. Gioja. 
Dasuki, S. (2010). Cuatro versiones de la ética y la moral. Bolivariana, Universidad Pontificia Medellín Colombia.

Gachama Muñoz, J. (2010). La ética kantiana y los postulados de la razón como inconsistencia de la filosofía crítica. Bajo Palabra. Revista de Filosofía.

García San Miguel, L. (2006). I. Kant: "Metafísica de las costumbres.” Filosofía Política. https://doi.org/10.1400/195196

González Vallejos, M. (2012). Factum de la razón y conciencia moral Acerca de la normatividad en la moral kantiana. Veritas. https://doi.org/10.4067/s071892732012000200006

Habermas, J. (1997). La idea kantiana de paz perpetua. Desde la distancia histórica de doscientos años. Isegoría. https://doi.org/10.3989/isegoria.1997.i16.184

Kant, Immanuel (1985). Fundamentación de la metafísica de las costumbres. Madrid. EspasaCalpe.

Mauricio, M. L. (2007). Concepto de la buena voluntad. Logos - Revista de La Facultad de Filosofía y Letras.

Mockus Sivickas, A. (1994). Anfibios culturales, moral y productividad. Revista Colombiana de Psicología.

Norbert, B. (1992). ¿Cómo Pensar los Valores Morales a Partir de Kant? Anales Del Seminario de Historia de La Filosofía. 
Placencia, L. (2013). Kant y la voluntad como "razón práctica". Kant and the will as practical reason. Tópicos, Revista de Filosofía. https://doi.org/10.21555/top.v41i1.75

Rivera Castro, F. (2004). El imperativo categórico en la fundamentación de la metafísica de las costumbres. Revista Digital Universitaria.

Román Maestre, B. (2007). La fundamentación kantiana del deber de vivir y el principio autonomía: reflexiones para la bioética. Laguna: Revista de Filosofía. 